\title{
Estrutura e atividades dos Núcleos de Segurança do Paciente em hospitais: uma revisão integrativa
}

\section{Structure and activities of Patient Safety Centers in hospitals: an integrative review}

\author{
Shaiane Coslop ${ }^{1, *}$ i \\ Bárbara do Nascimento Caldas" iD \\ Mariana Santana Rosário \\ Pereira' iD \\ Monalizza de Souza Carvalho \\ Calazans' iD
}

Eliane de Fátima Almeida Lima' (iD

Flávia Batista Portugal'

Programa de Pós-graduação em Saúde Coletiva (PPGSC), Universidade Federal do Espírito Santo (UFES), Vitória, ES, Brasil

" Instituto Nacional de Cardiologia (INC), Ministério da Saúde (MS), Rio de Janeiro, RJ, Brasil

\section{* E-mail: shaiane.saude@gmail.com}

Recebido: 06 jun 2021 Aprovado: 02 fev 2022

\begin{abstract}
RESUMO
Introdução: A partir da vigência do Programa Nacional de Segurança do Paciente (PNSP) no Brasil, tornou-se obrigatória a implantação de Núcleos de Segurança do Paciente (NSP) nos estabelecimentos de saúde. Apesar dos estudos sobre a implantação dos NSP em hospitais, até o momento, não há uma síntese que apresente pontos para orientar ações para melhoria de sua efetividade. Objetivo: Sintetizar o conhecimento disponível em publicações científicas brasileiras sobre a estruturação, funcionamento e atuação de NSP em hospitais. Método: Revisão integrativa de literatura realizada a partir da questão: "O que a produção científica apresenta sobre a estruturação e funcionamento de NSP em hospitais?". As buscas foram realizadas em junho de 2020, nas bases de dados LILACS, MEDLINE e BDENF, por meio de combinações dos seguintes descritores: Segurança do Paciente; Gestão de Riscos, Gestão de Segurança e Hospitais. Resultados: Doze artigos foram selecionados para inclusão. No geral, os NSP nos hospitais atendem parcialmente às diretrizes do PNSP. Foram identificadas falhas relacionadas às estruturas materiais e de recursos humanos dos NSP, bem como em relação às suas atividades. Aspectos do contexto local que representaram barreiras ou facilitadores para atuação efetiva dos NSP incluem questões materiais (por exemplo, dimensionamento e treinamento da equipe), simbólicas (por exemplo, cultura punitiva), relacionais (por exemplo, apoio da alta gestão) e institucionais (por exemplo, ferramentas para gestão da qualidade). Conclusões: A implantação de NSP nos hospitais tem apresentado avanços, mas ainda há muitos desafios para sua atuação efetiva. Melhorar a efetividade dos NSP exige criar um contexto mais favorável para o desenvolvimento de ações de melhoria da segurança.
\end{abstract}

PALAVRAS-CHAVE: Segurança do Paciente; Gestão de Riscos; Gestão de Segurança; Hospitais

\section{ABSTRACT}

Introduction: Since the establishment of the National Patient Safety Program (NPSP) in Brazil, the implementation of Patient Safety Centers (NSP) in health establishments has become mandatory. Although many studies on implementation of the NSP in hospitals have been published, until now, there is not any synthesis of the literature to inform how to improve NSP's effectiveness. Objective: To synthesize the knowledge of Brazilian scientific publications on the structuring and functioning of patient safety centers in Brazilian hospitals. Method: Integrative literature review based on the guiding question: "What does scientific production present about the structuring and functioning of NSP in hospitals?". The searches were carried out in June 2020, in the LILACS, MEDLINE and BDENF databases, through services of the following descriptors: Patient Safety; Risk Management, Safety Management and Hospitals. Results: Twelve studies were selected for inclusion. In general, NSP partially comply with NPSP's guidance. Failures related to NSP material and human resources structures were identified, as well as in relation to the NSP activities. Local contextual aspects that represented barriers or facilitators for the effective activity of NSP comprise: material (e.g., staffing and training), symbolic 
(e.g., culture of blame), relational (e.g., senior management support) and institutional (e.g., quality improvement tools) issues. Conclusions: NSP implementation at hospitals have presented some achievements. Improving NSP effectivity requires creating a supportive context for safety improvement efforts.

KEYWORDS: Patient Safety; Risk management; Safety Management; Hospitals

\section{INTRODUÇÃO}

Garantir a segurança do paciente (ou seja, redução do risco de dano desnecessário associado ao cuidado de saúde ${ }^{1}$ ) é um desafio permanente para os sistemas de saúde. Com o intuito de estabelecer estratégias para melhoria da qualidade do cuidado, o Ministério da Saúde instituiu, em 2013, o Programa Nacional de Segurança do Paciente (PNSP)2. Buscando estimular uma abordagem sistêmica para melhoria da segurança do paciente, as ações do PNSP foram planejadas em quatro grandes áreas: (1) atividades nos serviços de saúde; (2) envolvimento do cidadão; (3) educação e (4) pesquisa ${ }^{3}$. As atividades para segurança do paciente nos serviços de saúde foram instituídas pela Resolução da Diretoria Colegiada (RDC) $n^{\circ} 36$, de 25 de julho, da Agência Nacional de Vigilância Sanitária (Anvisa), que tornou obrigatória a implantação de Núcleos de Segurança do Paciente (NSP) nos estabelecimentos de saúde, exceto consultórios individualizados, laboratórios clínicos e os serviços móveis e de atenção domiciliar ${ }^{4}$.

Os NSP são instâncias responsáveis pelo desenvolvimento de estratégias para melhoria da qualidade do cuidado e pelo gerenciamento dos riscos assistenciais dentro dos serviços de saúde ${ }^{4}$. Cabe aos NSP a implementação de processos de identificação, análise, avaliação e monitoramento dos riscos, com posterior comunicação a todos os envolvidos ${ }^{4}$. Outras funções incluem: fortalecimento da cultura de segurança, a implantação de Planos de Segurança do Paciente (PSP) de acordo com a realidade local e a notificação de eventos adversos no sistema nacional ${ }^{4}$.

Os PSP contêm a padronização das ações estabelecidas pelos NSP para prevenção e gerenciamento dos riscos no processo de cuidado dos pacientes dentro dos estabelecimentos de saúde ${ }^{3}$. Dentre elas estão os protocolos básicos de segurança do paciente, que compreendem as estratégias para a identificação correta de pacientes, a realização de procedimentos cirúrgicos de maneira segura, a prática correta de higiene de mãos, a prevenção de lesões por pressão ${ }^{5}$ a prevenção de quedas e a segurança no uso, administração e prescrição de medicamentos ${ }^{6}$. Além destes, os PSP também devem possuir protocolos para segurança na prescrição, uso e administração de hemocomponentes, uso seguro de equipamentos e materiais, envolvimento de pacientes e familiares na assistência, promoção de ambiente seguro, promoção de comunicação efetiva entre os profissionais e entre serviços de saúde, segurança no uso de órteses e próteses e uso seguro de terapias nutricionais enterais e parenterais, e prevenção e controle de eventos adversos (incluindo infecções relacionadas à assistência à saúde) $)^{4}$.

A implementação do PNSP nos serviços de saúde tem sido lenta. Segundo dados da Anvisa, em novembro a maioria destes (4.285) situados em hospitais ${ }^{7}$. Oito anos após a publicação da RDC $n^{\circ} 36 / 2013$, dos 7.196 hospitais registrados no Cadastro Nacional de Estabelecimentos de Saúde $(\mathrm{CNES})^{8}$, aproximadamente $60 \%$ haviam estabelecido o NSP.

O cenário para a segurança do paciente nos hospitais brasileiros é bastante desafiador, incluindo déficit de recursos humanos, limitações relacionadas à notificação de eventos adversos, problemas nos programas de capacitação permanente, ausência de processos padronizados para o atendimento de pacientes, dentre outros 9 . 0 déficit de recursos materiais e humanos pode interferir no trabalho dos profissionais de enfermagem ${ }^{10}$, além de impactar negativamente o desenvolvimento de estratégias de segurança ${ }^{11}$ e os processos de trabalho interno dos NSP4.

Nos últimos anos, diversos estudos focando a estrutura e as atividades dos NSP em hospitais foram publicados ${ }^{12,13}$. Entretanto, um panorama geral ainda está faltando. De modo a propor ações para melhorar o desempenho dos NSP nos hospitais, é necessária uma compreensão mais detalhada sobre essa questão.

Esta revisão integrativa teve por objetivo sintetizar o conhecimento disponível em publicações científicas brasileiras sobre a estruturação, o funcionamento e a atuação dos NSP em hospitais.

\section{MÉTODO}

Trata-se de uma revisão integrativa, que consiste em uma síntese das informações disponíveis em dado momento, sobre um problema específico, de forma objetiva e reproduzível. Esse tipo de pesquisa apresenta um método de busca e seleção de estudos, com avaliação da relevância e da validade dos resultados evidenciados, coleta, síntese e interpretação dos dados. Dessa forma, utilizou-se um protocolo, a fim de garantir o rigor do processo de pesquisa, que dispunha dos seguintes componentes: pergunta de revisão, critérios de inclusão e exclusão, estratégias para a busca, orientação para a seleção do material, análise e síntese dos dados. 0 método foi escolhido, pois permite a síntese de estudos com mais de uma abordagem, proporcionando enfoque mais abrangente da situação temática ${ }^{14}$.

A pesquisa foi conduzida a partir da seguinte pergunta norteadora: "O que a literatura científica apresenta sobre a estruturação e funcionamento de Núcleos de Segurança do Paciente (NSP) em hospitais?". A busca foi realizada por dois pesquisadores em junho de 2020 nas bases de dados: Medical Literature Analysis and Retrieval System Online (MEDLINE), Literatura Latino-Americana e do Caribe em Ciências da Saúde (LILACS) e Base de Dados de Enfermagem (BDENF). Utilizou-se os seguintes descritores cadastrados nos Descritores em Ciências da Saúde (DeCS) e Medical Subject 
Headings (MeSH): "Patient Safety", "Risk Management", "Safety Management" e "Hospitals"; além das palavras-chave "Brazil" e "Brazilian", em português e inglês. O descritor "Patient Safety" foi utilizado como principal, combinado com cada um dos demais descritores com operador booleano "AND".

Foram incluídos artigos originais, publicados em espanhol, inglês ou português e cujo texto completo estivesse disponível. Optou-se pela inclusão de artigos originais, pois deseja-se um panorama da situação dos núcleos do Brasil após a publicação da RDC $n^{\circ} 36 / 2013$. Estudos publicados com coleta de dados anterior a novembro de 2013, início da obrigatoriedade da implantação de NSP em estabelecimentos de saúde, foram excluídos.

A seleção dos artigos foi feita em três etapas pelos pesquisadores: primeiro realizou-se a análise do título, após a do resumo e, por fim, a do artigo completo.

Os artigos selecionados constituíram o corpus da análise. Dados relevantes foram extraídos de cada artigo com base em um instrumento elaborado para pesquisa, incluindo base de dados, idioma, autores, título, metodologia, resultados (ou achados), implicações, nível de evidência (variando de 1 a 6 , sendo 1 a melhor evidência) e limitações identificadas ${ }^{14}$.

Os achados dos artigos foram analisados de modo descritivo visando caracterizar a estrutura e as atividades desenvolvidas pelos NSP a partir das diretrizes estabelecidas na RDC $n^{\circ}$ 36/2013. Adicionalmente, foram identificados as barreiras e os facilitadores para o desenvolvimento de ações de segurança do paciente pelos NSP. Estes foram posteriormente agrupados segundo quatro dimensões do contexto social, a saber: material (ou seja, oportunidades concretas de as pessoas colocarem em prática suas habilidades e iniciativas), simbólica (ou seja, significados e visões de mundo), relacional (ou seja, processos de liderança, trabalho em equipe) e institucional (ou seja, estruturas e mecanismos para segurança) ${ }^{15}$.

\section{RESULTADOS}

Foram encontrados 2.556 artigos, 382 provenientes da base de dados MEDLINE, 1.511 da LILACS e 663 da BDENF. Após a aplicação dos critérios de inclusão e exclusão, foram selecionados 13 artigos. Um artigo foi excluído por apresentar dados semeIhantes com outro, ou seja, foram realizados no mesmo hospital e apresentaram metodologia, resultados e discussão similares. Ao final, 12 artigos foram selecionados (Figura). Ressalta-se que cinco artigos corresponderam a produtos de duas pesquisas. Os resultados corresponderam, portanto, a nove pesquisas e/ou relatos de experiência exclusivos.

Em relação ao delineamento dos estudos, cinco são descritivos qualitativos, cinco quantitativos e dois relatos de experiência (Quadro). Os dados dos artigos qualitativos foram coletados por

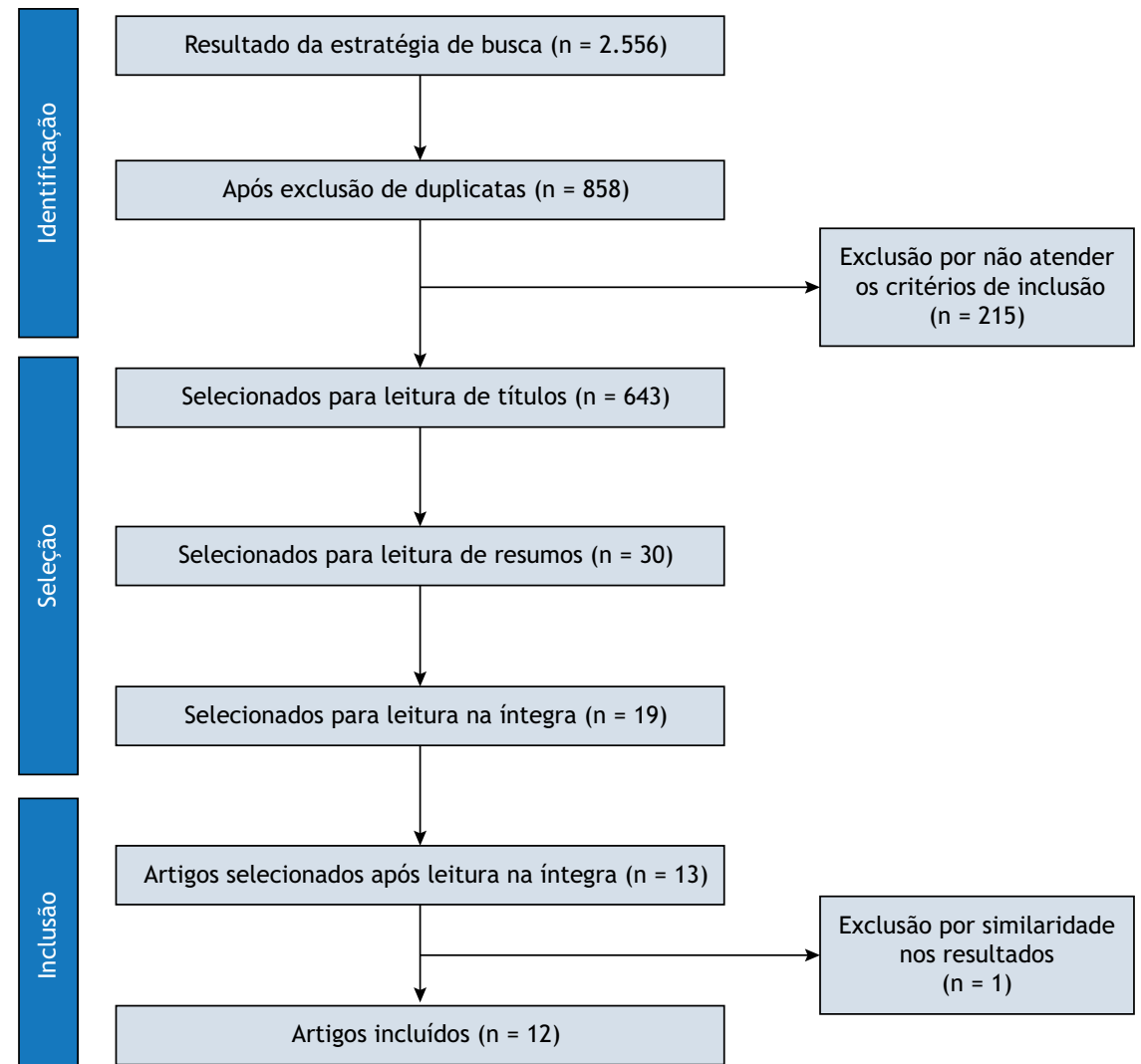

Fonte: Elaborada pelos autores, 2020.

Figura. Fluxograma de seleção de artigos. 
entrevista semiestruturada ${ }^{12,16,17,18,19}$, análise documental ${ }^{12,19} \mathrm{e}$ observação não participante ${ }^{12,19}$, e abordaram a perspectiva de profissionais sobre fatores que influenciaram a implementação de estratégias de segurança. Já os quantitativos foram coletados por meio de entrevistas ${ }^{20,21,22,23}$, observação direta ${ }^{20}$, verificação de registros ${ }^{20}$, sendo informado que a coleta de dados foi realizada com o mesmo instrumento validado em dois artigos ${ }^{13,22}$. Estes estudos abordaram a caracterização da estrutura e atividades dos NSP. $O$ conjunto de artigos selecionado apresenta baixo nível de evidência, por se tratar em sua maioria de estudos descritivos (nível 4).

Os dados foram coletados em hospitais das regiões Sudeste (quatro artigos, 14 hospitais), Centro-oeste (um artigo, seis hospitais), Sul (cinco artigos, cinco hospitais) e Nordeste (dois artigos, 24 hospitais) do Brasil, correspondendo a dados de hospitais públicos $(n=32)$, filantrópicos $(n=11)$, privados $(n=6)$ e de parceria público-privada $(\mathrm{n}=1)$.

A síntese dos achados dos artigos selecionados para a revisão foi organizada em dois temas/tópicos: estrutura e atividades dos NSP, e barreiras e facilitadores para o desenvolvimento de ações de segurança do paciente.

\section{Estrutura e atividades dos NSP}

A maioria dos trabalhos aponta que os NSP apresentavam limitações relacionadas às suas estruturas e processos de trabalho.

Quadro. Título, delineamento da pesquisa/participantes e principais achados dos artigos incluídos na revisão.

\begin{tabular}{|c|c|c|c|}
\hline Autores (ano) & Título & Delineamento/participantes & Principais achados \\
\hline $\begin{array}{l}\text { Cavalcante et al. } \\
(2019)^{21}\end{array}$ & $\begin{array}{l}\text { Implementação dos núcleos } \\
\text { de segurança do paciente e } \\
\text { as infecções relacionadas à } \\
\text { assistência à saúde }\end{array}$ & $\begin{array}{l}\text { Quantitativo transversal/ } \\
12 \text { hospitais, } 28 \text { profissionais } \\
\text { controladores de infecção, } \\
\text { dados coletados por entrevista }\end{array}$ & $\begin{array}{c}\text { A maioria dos hospitais possuía NSP e os protocolos de } \\
\text { higiene de mãos, identificação do paciente, prevenção de } \\
\text { quedas foram os mais implantados. }\end{array}$ \\
\hline $\begin{array}{l}\text { Costa et al. } \\
(2020)^{23}\end{array}$ & $\begin{array}{l}\text { Segurança do paciente em } \\
\text { serviços de saúde: uma análise } \\
\text { na cidade de Salvador, Bahia }\end{array}$ & $\begin{array}{l}\text { Quantitativo/membros do NSP de } \\
12 \text { hospitais de grande porte }\end{array}$ & $\begin{array}{l}\text { Os hospitais estudados possuíam NSP e a maioria havia } \\
\text { implantado todos os protocolos básicos. Fragilidades em } \\
\text { processos de capacitação na área de segurança do paciente. }\end{array}$ \\
\hline $\begin{array}{l}\text { Macedo e } \\
\text { Bohomol (2019) }\end{array}$ & $\begin{array}{l}\text { Análise da estrutura } \\
\text { organizacional do núcleo de } \\
\text { segurança do paciente dos } \\
\text { hospitais da Rede Sentinela }\end{array}$ & $\begin{array}{l}\text { Quantitativo exploratório e } \\
\text { descritivo/12 hospitais, } \\
12 \text { coordenadores de NSP, } \\
\text { dados coletados por } \\
\text { autopreenchimento de formulário }\end{array}$ & $\begin{array}{l}\text { As instituições possuíam NSP. } \\
\text { Fragilidades foram encontradas em processos dos NSP, } \\
\text { gestão de risco, nos programas de capacitação permanente } \\
\text { e na disponibilidade de recursos. }\end{array}$ \\
\hline $\begin{array}{l}\text { Macedo et al. } \\
(2018)^{22}\end{array}$ & $\begin{array}{l}\text { Implantação do núcleo de } \\
\text { segurança do paciente em } \\
\text { hospital universitário }\end{array}$ & $\begin{array}{l}\text { Quantitativo descritivo/ } \\
\text { um hospital, dados coletados } \\
\text { por entrevista }\end{array}$ & $\begin{array}{l}\text { NSP implantado, havia apoio da alta direção, estrutura } \\
\text { física e recursos humanos adequados. } \\
\text { Era realizado gerenciamento de riscos para os protocolos. } \\
\text { Não estavam implantados os protocolos de comunicação } \\
\text { segura e de envolvimento dos pacientes em sua segurança. }\end{array}$ \\
\hline $\begin{array}{l}\text { Oliveira et al. } \\
(2017)^{18}\end{array}$ & $\begin{array}{l}\text { Fatores facilitadores na } \\
\text { implantação das estratégias de } \\
\text { segurança do paciente: estudo } \\
\text { descritivo-exploratório }\end{array}$ & $\begin{array}{l}\text { Qualitativo exploratório } \\
\text { descritivo/quatro hospitais, } \\
72 \text { enfermeiros gestores }\end{array}$ & $\begin{array}{l}\text { Foram apontados como facilitadores o apoio da alta direção } \\
\text { e gestores dos setores, a realização de gestão de riscos, } \\
\text { de processos de educação permanente. }\end{array}$ \\
\hline $\begin{array}{l}\text { Prates et al. } \\
(2019)^{24}\end{array}$ & $\begin{array}{l}\text { Núcleo de segurança do } \\
\text { paciente: o caminho das pedras } \\
\text { em um hospital geral }\end{array}$ & $\begin{array}{l}\text { Relato de experiência/um } \\
\text { hospital, análise documental e } \\
\text { relato de experiência das autoras }\end{array}$ & $\begin{array}{l}\text { Implementadas ações para fortalecimento de cultura } \\
\text { de segurança, implementados os protocolos básicos, } \\
\text { gerenciamento de riscos e de incidentes e o monitoramento } \\
\text { dos indicadores de segurança do paciente. }\end{array}$ \\
\hline $\begin{array}{l}\text { Reis et al. } \\
(2019)^{16}\end{array}$ & $\begin{array}{l}\text { Dificuldades para implantar } \\
\text { estratégias de segurança do } \\
\text { paciente: perspectivas de } \\
\text { enfermeiros gestores }\end{array}$ & $\begin{array}{l}\text { Qualitativo, exploratório } \\
\text { descritivo/quatro hospitais, } \\
72 \text { enfermeiros gestores }\end{array}$ & $\begin{array}{l}\text { Dificuldades compreenderam déficit de pessoal de } \\
\text { enfermagem, baixa adesão de profissionais assistenciais. }\end{array}$ \\
\hline $\begin{array}{l}\text { Reis et al. } \\
(2017)^{17}\end{array}$ & $\begin{array}{l}\text { Nurse manager perceptions } \\
\text { of patient safety strategy } \\
\text { implementation }\end{array}$ & $\begin{array}{l}\text { Qualitativo exploratório } \\
\text { descritivo/quatro hospitais, } \\
72 \text { enfermeiros gestores }\end{array}$ & $\begin{array}{l}\text { O atraso na implantação ocasionou sentimentos de } \\
\text { decepção em alguns profissionais. } \\
\text { Outros sentiam satisfação por estarem envolvidos nas } \\
\text { estratégias de segurança. }\end{array}$ \\
\hline $\begin{array}{l}\text { Santos et al. } \\
(2019)^{25}\end{array}$ & $\begin{array}{l}\text { Avaliação da implantação } \\
\text { de um núcleo de segurança } \\
\text { do paciente }\end{array}$ & $\begin{array}{l}\text { Relato de experiência/um NSP } \\
\text { de um hospital }\end{array}$ & $\begin{array}{l}\text { O NSP implementou ações para protocolos de higiene de } \\
\text { mãos, cirurgia segura, identificação do paciente, } \\
\text { e prevenção de lesões por pressão. }\end{array}$ \\
\hline $\begin{array}{l}\text { Serra et al. } \\
(2016)^{20}\end{array}$ & $\begin{array}{l}\text { Situação dos hospitais de } \\
\text { referência para implantação/ } \\
\text { funcionamento do núcleo de } \\
\text { segurança do paciente }\end{array}$ & $\begin{array}{l}\text { Quantitativo descritivo/seis } \\
\text { hospitais, dados coletados por } \\
\text { observação direta e verificação } \\
\text { de registros }\end{array}$ & $\begin{array}{l}\text { Um hospital não possuía NSP. Identificados problemas como: } \\
\text { escassez de profissionais, equipamentos e materiais. }\end{array}$ \\
\hline $\begin{array}{l}\text { Siman e Brito } \\
(2018)^{12}\end{array}$ & $\begin{array}{l}\text { A dimensão prescrita e real } \\
\text { de práticas de profissionais } \\
\text { de saúde no contexto da } \\
\text { segurança do paciente }\end{array}$ & $\begin{array}{l}\text { Qualitativo do tipo estudo } \\
\text { de caso/um hospital, } \\
31 \text { participantes membros } \\
\text { dos NSP e enfermeiros, dados } \\
\text { saturados com } 12 \text { entrevistas }\end{array}$ & $\begin{array}{c}\text { Havia distância entre protocolos prescritos e a realidade, } \\
\text { déficit de recursos humanos, materiais e falhas em } \\
\text { programa de capacitação continuada. }\end{array}$ \\
\hline $\begin{array}{l}\text { Siman et al. } \\
(2019)^{19}\end{array}$ & $\begin{array}{l}\text { Desafios da prática na } \\
\text { segurança do paciente }\end{array}$ & $\begin{array}{l}\text { Qualitativo do tipo estudo } \\
\text { de caso/31 profissionais de } \\
\text { um hospital }\end{array}$ & $\begin{array}{c}\text { Existiam NSP e protocolos prescritos e dificuldades para } \\
\text { implementar as ações. Déficits de recursos humanos e } \\
\text { materiais constituíam desafios. }\end{array}$ \\
\hline
\end{tabular}

Fonte: Elaborado pelos autores, 2020.

NSP: Núcleo de Segurança do Paciente. 
Destacavam-se problemas na disponibilidade de equipamentos básicos ${ }^{12,20,23}$, ausência de espaço e equipamentos dedicados aos NSP $^{13,21,23}$ e escassez de recursos para a implementação de ações do PSP13,19,20,23. A carência de profissionais e de pessoal capacitado na área de qualidade ou de segurança do paciente ${ }^{13,19,20,23}$ foi um achado frequente, além da ausência de profissionais com dedicação exclusiva ao trabalho no NSP23. Enfermeiros ${ }^{12,19,21,23}$, farmacêuticos ${ }^{12,19,21,23}$ e médicos ${ }^{21,23}$ eram os profissionais que com maior frequência compunham o NSP. Ainda assim, apresentou-se relevante o número de NSP sem a presença de médicos ${ }^{12,19,22,23,24}$.

Em relação às atividades, os estudos apontaram fragilidades na gestão de processos ${ }^{20}$, problemas de comunicação entre as equipes ${ }^{12,21}$ e ausência da realização de notificações de eventos adversos, tanto internamente ${ }^{23}$ como no sistema nacional ${ }^{13}$.

A implantação dos protocolos estabelecidos pela RDC n 36/2013 nos hospitais mostrou-se variável. Os protocolos implantados com maior frequência foram: identificação do paciente, higienização de mãos, prevenção de quedas ${ }^{13,21,22,23}$, controle de infecções relacionadas à assistência à saúde, cirurgia segura, prevenção de lesão por pressão e registro de órteses ${ }^{13,21,22}$. Os demais protocolos - comunicação efetiva ${ }^{21,22,25}$, segurança na prescrição, uso e administração de medicamentos ${ }^{21}$, envolvimento do paciente em sua segurança ${ }^{21,22}$, segurança em nutrição enteral e parenteral, segurança na transfusão sanguínea e uso seguro de equipamentos e materiais ${ }^{13,22}$ - foram implantados por um número menor de NSP. Em alguns hospitais, a implantação dos protocolos foi acompanhada do monitoramento dos seus indicadores ${ }^{21,23}$. Ainda com relação aos protocolos, alguns artigos destacaram a distância entre o que estava formalizado nos documentos institucionais e as práticas que realmente eram executadas pelas equipes assistenciais $^{12,19}$.

A capacitação dos profissionais em qualidade e segurança do paciente, estratégia fundamental para melhoria da segurança, recebeu pouca atenção por parte dos NSP. Nem todos os hospitais promoviam ações de capacitação ${ }^{12}$, enquanto outros as realizavam de forma deficitária, faziam somente na admissão dos profissionais ao estabelecimento ${ }^{13}$ ou não abrangiam todos os profissionais ${ }^{20}$. Mesmo os hospitais que desenvolvem ações de capacitação convivem com fragilidades nas atividades relacionadas à segurança do paciente, como a não participação ativa da equipe de enfermagem assistencial na gestão e vigilância dos riscos ${ }^{21}$.

Barreiras e facilitadores para o desenvolvimento de ações de segurança do paciente pelos Núcleos de Segurança do Paciente

As ações de segurança do paciente, coordenadas pela equipe do NSP, sofrem influência de aspectos do contexto local que podem atuar como barreiras ${ }^{12,16,19,20,21,24}$ ou facilitadores ${ }^{17,18,24}$ ao seu desenvolvimento. Barreiras relacionadas ao contexto material identificadas nos estudos incluíam falta de capacitação na área de segurança do paciente ${ }^{12,24}$, equipes assistenciais subdimensionadas, sobrecarga de trabalho, problemas de remuneração, estresse, rotatividade, condições estruturais precárias em locais de internação, materiais insuficientes e de má qualidade, ausência de manutenção e/ou ausência de equipamentos ${ }^{19}$. Esses pontos prejudicavam tanto a estruturação e o trabalho interno do NSP quanto as ações que o NSP desenvolvia junto às equipes assistenciais, como a implementação dos protocolos, e, em alguns hospitais, foram atribuídas à escassez de recursos financeiros $^{20}$ e materiais ${ }^{21}$.

Com relação aos aspectos simbólicos e relacionais do contexto foram consideradas como barreiras ao avanço das ações: o baixo apoio da alta direção ${ }^{16}$, a existência da cultura punitiva dentro dos hospitais e a resistência de alguns profissionais da saúde em aderir aos processos de segurança ${ }^{12,24}$. A resistência da equipe assistencial, manifestada pela não adesão às práticas de segurança, foi referida como causa de desmotivação em profissionais que geriam as ações ${ }^{16}$. Outro fator de desmotivação de enfermeiros gestores relacionou-se à frustração por perceber as ações em seu hospital em atraso ao compará-lo a outros hospitais que iniciaram seus NSP no mesmo período ${ }^{18}$.

Aspectos facilitadores para o desenvolvimento de ações de segurança do paciente nos hospitais foram apresentados por um número menor de artigos. Nas dimensões simbólica e relacional, incluíram apoio da alta direção e a existência de lideranças comprometidas ${ }^{18}$; na dimensão institucional, processos de acreditação hospitalar implantados ${ }^{17}$, e o uso de ferramentas da qualidade e de gestão de risco na rotina de trabalho ${ }^{18,24}$; e na dimensão material, a realização de capacitações contínuas ${ }^{18}$. Um fator motivador para as equipes envolvidas na implantação de novas práticas foi a satisfação causada pelo impacto positivo das ações realizadas ${ }^{17}$.

\section{DISCUSSÃO}

Este artigo apresenta os achados de uma revisão da literatura sobre a estrutura e as atividades dos NSP em hospitais, componente da área "atividades nos serviços de saúde" do PNSP. A revisão da literatura permitiu identificar que os NSP nos hospitais atendem em parte às diretrizes da RDC Anvisa $n^{\circ}$ 36/2013. Muitos dos NSP formalmente estabelecidos têm dificuldades para avançar nas atividades propostas por não encontrarem um contexto favorável ao desenvolvimento de ações para melhoria da segurança do paciente. As barreiras identificadas vão desde a falta de apoio da alta direção, subdimensionamento das equipes, capacitação insuficiente, equipamentos e insumos inadequados, até cultura punitiva e resistência por parte das equipes assistenciais às ações propostas pelos NSP. Importante destacar que muitas das barreiras identificadas impactam tanto o NSP quanto as equipes assistenciais. Os facilitadores identificados por esta revisão correspondem ao lado oposto da moeda, como o apoio da alta direção, as lideranças comprometidas, a adoção consistente de práticas de qualidade e gestão do risco, e as capacitações adequadas.

A melhoria do sistema em relação à segurança do paciente nas organizações de saúde envolve questões culturais, estruturais e de processos ${ }^{1}$. A alta direção tem papel imprescindível na melhoria do sistema, uma vez que seu envolvimento nas ações de segurança pode gerar resultados positivos no clima de segurança, 
no engajamento das equipes e nos resultados das ações de segurança ${ }^{26}$. Características de líderes de organizações seguras incluem o olhar sistêmico sobre os problemas de segurança e a incorporação de práticas voltadas para minimização de riscos ${ }^{27}$. É papel da direção a disponibilização adequada de recursos humanos, equipamentos, materiais e recursos financeiros ${ }^{4}$, além de atuação proativa no estabelecimento de metas e iniciativas voltadas à segurança ${ }^{28}$.

É importante que a alta direção utilize uma visão racional ao estruturar as equipes de NSP, pois esses profissionais precisam desempenhar um papel de articulação dentro do serviço de saúde $^{3,29}$. Para tanto, é ideal que a composição dessas equipes seja multiprofissional, incluindo minimamente farmacêuticos, médicos e enfermeiros ${ }^{29}$. Essa composição mínima não foi relatada em muitos dos NSP estudados. Esse fato pode ter contribuído nas fragilidades de processo encontradas nos NSP estudados, tais como os problemas no processo de notificação (interna e externa) e a falta de robustez na implementação prática dos protocolos de segurança.

A maioria dos protocolos que foram considerados implantados correspondem aos protocolos básicos, com exceção dos protocolos de controle de infecções, cujas ações mínimas foram estabelecidas em período anterior à da segurança do paciente, em $1998^{30}$. A diferença existente entre o tempo da obrigatoriedade de implantação dos protocolos de controle de infecções e os protocolos de segurança do paciente (aproximadamente 15 anos) pode ter favorecido os maiores resultados positivos encontrados a favor do controle de infecção.

A constatação de que protocolos de segurança do paciente podem se expressar mais em sua dimensão prescrita do que real (ou seja, existir de forma documental escrita, mas não ser executado na prática $)^{12}$ demonstra a necessidade de os NSP conhecerem melhor como as equipes assistenciais colocam em prática os protocolos. Essa abordagem permite saber quais etapas do protocolo têm menor adesão pelas equipes e porque isso ocorre, e pode orientar o planejamento, em conjunto com os profissionais da ponta, de iniciativas para aumentar a adesão.

Uma das contribuições para a baixa adesão aos protocolos de segurança parece estar relacionada ao dimensionamento das equipes assistenciais ${ }^{11,31}$. A alta carga de trabalho pode contribuir para que os profissionais tenham maior percepção de estresse e maior probabilidade de gerar prejuízos à qualidade do cuidado ao paciente $^{32}$. Além disso, condições ruins de trabalho e remuneração, conflitos internos nas equipes e conflitos entre profissionais e instituição podem gerar esgotamento ${ }^{33}$ e contribuir com a baixa adesão dos profissionais e subnotificação de eventos adversos ${ }^{34}$.

Implementar estratégias efetivas para o aumento da adesão às práticas seguras é um desafio, pode compreender estratégias educativas $^{35}$ e de comunicação efetiva ${ }^{36}$ e requer a participação ativa dos NSP4 . Engloba ações de incentivo constante à participação dos profissionais nos processos e de fortalecimento da cultura justa, uma vez que processos de trabalho que não promovem a inclusão dos profissionais podem estar associados a baixas taxas de notificação de eventos adversos ${ }^{34}$. Além disso, a implementação de ações voltadas à promoção de boas relações dentro das equipes pode contribuir para que os profissionais possuam uma menor percepção de sobrecarga no trabalho ${ }^{37}$.

As estratégias de educação permanente têm papel importante na evolução da percepção positiva sobre a segurança do paciente ${ }^{35,38}$. Entretanto, mesmo hospitais que desenvolvem ações de capacitação convivem com fragilidades nas atividades relacionadas à segurança do paciente e na participação ativa da equipe de enfermagem assistencial na gestão e vigilância dos riscos ${ }^{21}$. Esse achado sugere que mesmo que realizadas de modo adequado, as ações de capacitação não geram resultados imediatos. Reforça também a noção de que, embora seja uma estratégia fundamental, a capacitação das equipes não é suficiente para garantir adesão a práticas seguras ${ }^{26}$.

Os resultados apresentados apontam para a necessidade ainda atual de fortalecimento da cultura de segurança dentro dos hospitais. Inclui nesse raciocínio a prática da cultura justa baseada no comportamento humano frente ao incidente, com distinção entre erro humano, comportamento de risco e imprudência, e adoção de visão sistemática da ocorrência desses eventos $^{4}$. O não enfrentamento da cultura punitiva pode ser fator agravante para o insucesso de ações de promoção de cultura de segurança ${ }^{39}$. 0 fortalecimento da cultura de segurança inclui a realização de ações voltadas à valorização dos profissionais, à implementação dos protocolos de segurança, ao apoio dos gestores e à promoção de boas condições de trabalho ${ }^{40,41}$. A incorporação dessas ações de melhoria demanda NSP ativos ${ }^{29}$, o que enfatiza a importância de sua estruturação adequada.

A diversidade metodológica dos artigos incluídos nesta revisão permitiu maior amplitude na identificação dos fatores relacionados ao objeto de estudo, configurando-se em um ponto forte do artigo. A revisão também foi abrangente com relação à natureza dos hospitais, incluindo estudos que apresentaram resultados referentes a hospitais públicos, filantrópicos e privados. Entretanto, a amostragem de estabelecimentos estudados foi relativamente pequena ao se considerar a totalidade e a diversidade de hospitais existentes no Brasil, o que constitui limitação desta revisão. Agrega-se o fato de que os achados não foram homogeneamente distribuídos por regiões geográficas e estados brasileiros. A região Norte do Brasil não foi contemplada em nenhum dos estudos. As regiões geográficas comportam diferenças em relação à disponibilidade de recursos hospitalares, concentrando maior número de hospitais de grande porte na região Sudeste ${ }^{42}$. Enquanto a região Norte concentra o menor número leitos de internação em relação às outras regiões ${ }^{43}$. Estudo relacionou a região Norte a menores índices de eficiência técnica hospitalar nos anos de 2014 e 2015, em comparação com as outras regiões do Brasil ${ }^{44}$. Tais fatos podem estar envolvidos na escassez de artigos publicados sobre NSP estruturados em hospitais na região. Outra limitação do presente estudo se refere ao baixo nível de evidência dos artigos incluídos na revisão.

Além disso, os resultados indicaram que o tema ainda é pouco explorado no meio científico, destacando a necessidade de 
realização de maior número de pesquisas sobre a temática no Brasil, inclusive com maior abrangência das regiões geográficas. Dessa forma, o conhecimento sobre como proporcionar atuação mais efetiva dos NSP em hospitais seria aprofundado.

Os achados desta revisão apontam questões que devem ser tratadas por formuladores de política de saúde, diretores de hospitais e equipes dos NSP de modo a favorecer os esforços para melhoria da segurança do paciente. Como ponto de partida, mais atenção deve ser dada ao adequado dimensionamento e à capacitação das equipes assistenciais e de gestão da qualidade e segurança. Adicionalmente, a conservação inadequada de equipamentos e a falta de insumos devem ser enfrentadas, pois estas, juntamente com o subdimensionamento de pessoal, afetam profundamente a capacidade e a motivação dos profissionais de saúde em fornecer um cuidado seguro ${ }^{45}$. Considerando o papel crucial da alta liderança em estimular e criar um contexto favorável à segurança do paciente, esforços visando o desenvolvimento e engajamento de diretores, coordenadores e gerentes também são necessários. Por fim, os desafios para obter adesão às práticas seguras exigem das equipes dos NSP formas de implantar os protocolos que vão além de redigir um documento e treinar a equipe. Mostram também a necessidade de adoção de lógicas de monitoramento dos protocolos que, mais que dados, gerem inteligência, privilegiando a busca ativa de fragilidades e o uso de múltiplos métodos - sem depender do preenchimento de formulários ${ }^{46}$.

Esta revisão teve como objetivo apresentar um panorama geral da estrutura e das atividades dos NSP em hospitais. Entre os artigos selecionados, poucos abordaram atividades relacionadas ao monitoramento de protocolos de segurança e ao envolvimento de pacientes e familiares em sua segurança. Estudos originais ou revisões da literatura focando atividades específicas são importantes para gerar conhecimento sobre práticas no cenário brasileiro e identificar lições aprendidas. Estudos que avaliam resultados das práticas implantadas também são fundamentais para melhor entendimento do assunto.

\section{CONCLUSÕES}

A implantação dos NSP nos hospitais tem apresentado avanços, mas ainda há muitos desafios para sua atuação efetiva. Melhorar a efetividade dos NSP exige criar um contexto mais favorável para o desenvolvimento de ações de melhoria da segurança. Abordar as barreiras identificadas neste estudo é um bom começo, reconhecendo, sobretudo, o papel fundamental da alta gestão na promoção da segurança do paciente como prioridade e na garantia do dimensionamento e treinamento adequados, tanto das equipes assistenciais quanto do NSP.

\section{REFERÊNCIAS}

1. Runciman $W$, Hibbert $P$, Thomson $R$, Van Der Schaaf T, Sherman H, Lewalle P. Towards an international classification for patient safety: key concepts and terms. Int J Qual Heal Care. 2009;21(1):18-26. https://doi.org/10.1093/intqhc/mzn057

2. Ministério da Saúde (BR). Portaria $N^{\circ} 529$, de 1 de abril de 2013. Institui o Programa Nacional de Segurança do Paciente (PNSP). Diário Oficial Uniao. 2 abr 2013.

3. Agência Nacional de Vigilância Sanitária - Anvisa. Documento de referência para o Programa Nacional de Segurança do Paciente. Brasília: Agência Nacional de Vigilância Sanitária; 2014.

4. Agência Nacional e Vigilância Sanitária - Anvisa. Resolução $\mathrm{RDC} \mathrm{N}^{\circ}$ 36, de 25 de julho de 2013. Institui ações para segurança do paciente em serviços de saúde. Diário Oficial Uniao. 26 jul 2013.

5. Ministério da Saúde (BR). Portaria $N^{\circ} 1.377$, de 9 de julho de 2013. Aprova os protocolos de segurança do paciente. Dário Oficial União. 10 jul 2013.

6. Ministério da Saúde (BR). Portaria $\mathrm{N}^{\circ} 2.095$, de 24 de setembro de 2013. Aprova os protocolos básicos de segurança do paciente. Diário Oficial Uniao. 25 set 2013.

7. Agência Nacional e Vigilância Sanitária - Anvisa. Núcleos de Segurança do Paciente. Brasília: Agência Nacional e Vigilância Sanitária; 2021[acesso 19 jun 2021]. Disponível em: https://www.gov.br/anvisa/pt-br/ acessoainformacao/dadosabertos/informacoes-analiticas/ nucleos-de-seguranca-do-paciente
8. Ministério da Saúde (BR). Datasus: cadastro nacional de estabelecimentos de saúde. Brasília: Ministério da Saúde; 2021 [acesso 10 maio 2021] . Disponível em: http://cnes. datasus.gov.br/

9. Sartor GD, Silva BF, Masiero AV. Segurança do paciente em hospitais de grande porte: panorama e desafios. Cogitare Enferm. 2016;21(5):1-8. https://doi.org/10.5380/ce.v21i5.45644

10. Siman AG, Brito MJM. Mudanças na prática de enfermagem para melhorar a segurança do paciente. Rev Gaúcha Enferm. 2017;37(esp):1-9. https://doi.org/10.1590/1983-1447.2016.esp.68271

11. Gama ZAS, Saturno-Hernández PJ, Ribeiro DNC, Freitas MR, Medeiros PJ, Batista AM et al. Development and validation of indicators for best patient safety practices: the ISEP-Brazil project. Cad Saúde Pública. 2016;32(9):1-17. https://doi.org/10.1590/0102-311X00026215

12. Siman AG, Brito MJM. A dimensão prescrita e real de práticas de profissionais de saúde no contexto da segurança do paciente. Rev Enferm Uerj. 2018;26:1-6. https://doi.org/10.12957/reuerj.2018.23703

13. Macedo RS, Bohomol E. Análise da estrutura organizacional do Núcleo de Segurança do Paciente dos hospitais da Rede Sentinela. Rev Gaúcha Enferm. 2019;40(esp):1-10. https://doi.org/10.1590/1983-1447.2019.20180264

14. Souza MT, Dias M, Carvalho R. Revisão integrativa : o que é e como fazer. Einstein. 2010;8(1):102-6. https://doi.org/10.1590/S1679-45082010RW1134 
15. Campbell C, Cornish F. How can community health programmes build enabling environments for transformative communication? Experiences from India and South Africa. AIDS Behav. 2012;16(4):847-57. https://doi.org/10.1007/s10461-011-9966-2

16. Reis GAX, Oliveira JLC, Ferreira AMD, Vituri DW, Marcon SS, Matsuda LM. Dificuldades para implantar estratégias de segurança do paciente: perspectivas de enfermeiros gestores. Rev Gaúcha Enferm. 2019;40(esp):1-7. https://doi.org/10.1590/1983-1447.2019.20180366

17. Reis GAX, Hayakawa LY, Murassaki ACY, Matsuda LM, Gabriel CS, Oliveira MLF. Nurse manager perceptions of patient safety strategy implementation. Texto Contexto Enferm. 2017;26(2):1-9. https://doi.org/10.1590/0104-07072017000340016

18. Oliveira JLC, Reis GAX, Souza VS, Costa MAR, Valera IMA, Matsuda LM. Fatores facilitadores na implantação das estratégias de segurança do paciente: estudo descritivo-exploratório. Online Braz J Nurs. 2017;16(2):108-18. https://doi.org/10.17665/1676-4285.20175515

19. Siman AGS, Braga LM, Amaro MOF, Brito MJM. Desafios da prática na segurança do paciente. Rev Bras Enferm. 2019;72(6):1581-8. https: //doi.org/10.1590/0034-7167-2018-0441

20. Serra JN, Barbieri AR, Cheade MFM. Situação dos hospitais de referência para implantação/funcionamento do Núcleo de Segurança do Paciente. Cogitare Enferm. 2016;21(5):1-9. https://doi.org/10.5380/ce.v21i5.45925

21. Cavalcante EFO, Pereira IRBO, Leite MJVF, Santos AMD, Cavalcante CAA. Implementação dos Núcleos de Segurança do Paciente e as infecções relacionadas à assistência à saúde. Rev Gaúcha Enferm. 2019;40(esp):1-10. https: / / doi.org/10.1590/1983-1447.2019.20180306

22. Macedo RS, Teixeira DV, Bohomo E. Implantação do Núcleo de Segurança do Paciente em hospital universitário. Nursing (São Paulo). 2018;21(246):2431-4.

23. Costa EAM, Lobão WM, Ribas CLM, Passos NM. Segurança do paciente em serviços de saúde: uma análise na cidade de Salvador, Bahia. Rev Sobecc. 2020;25(1):17-24. https: / / doi.org/10.5327/Z1414-4425202000010004

24. Prates CG, Magalhães AMM, Balen MA, Moura GMSS. Núcleo de Segurança do Paciente: o caminho das pedras em um hospital geral. Rev Gaúcha Enferm. 2019;40(esp):1-5. https: / / doi.org/10.1590/1983-1447.2019.20180150

25. Santos RP, Soppa FBF, Ruths JC, Rizzotto MLF. Avaliação da implantação de um Núcleo de Segurança do Paciente. Rev Enferm UFPE. 2019;13(2):532-7. https://doi. org/10.5205/1981-8963-v13i02a238189p532-537-2018

26. Singer SJ, Vogus TJ. Reducing hospital errors: Interventions that build safety culture. Annu Rev Public Health. 2013;34:373-96. https://doi.org/10.1146/ annurev-publhealth-031912-114439
27. Clarke JR, Lerner JC, Marella W. The role for leaders of health care organizations in patient safety. Am J Med Qual. 2007;22(5):311-8. https://doi.org/10.1177/1062860607304743

28. Moffatt-bruce S, Clark S, Dimaio M, Fann J. Leadership oversight for patient safety programs: an essential element. Ann Thorac Surg. 2018;105(2):351-6. https://doi.org/10.1016/j.athoracsur.2017.11.021

29. Agência Nacional de Vigilânica Sanitária - Anvisa. Implantação do Núcleo de Segurança do Paciente em serviços de saúde. Brasília: Agência Nacional de Vigilânica Sanitária; 2016[acesso 1 set 2020]. Disponível em: https://www.gov.br/anvisa/pt-br/centraisdeconteudo/ publicacoes/servicosdesaude/publicacoes

30. Ministério da Saúde (BR). Portaria $N^{\circ} 2.616$, de 12 de maio de 1998. Expede, na forma dos anexos I, II, III, IV, $\mathrm{V}$, diretrizes e normas para a prevenção e o controle das infecções hospitalares. Diário Oficial Uniao. 13 maio 1998.

31. Aiken LH, Clarke SP, Sloane DM, Sochalski J, Silber JH. Hospital nurse staffing and patient mortality, nurse burnout, and job dissatisfaction. JAMA. 2002;288(16):1987-93. https://doi.org/10.1001/jama.288.16.1987

32. Sturm H, Rieger MA, Martus P, Ueding E, Wagner A, Holderried $M$ et al. Do perceived working conditions and patient safety culture correlate with objective workload and patient outcomes: a cross-sectional explorative study from a German university hospital. PLoS One. 2019;14(1):1-19. https://doi.org/10.1371/journal.pone.0209487

33. Meneghini F, Paz AA, Lautert L. Fatores ocupacionais associados aos componentes da síndrome de burnout em trabalhadores de enfermagem. Texto Contexto Enferm. 2011;20(2):225-33. https://doi.org/10.1590/\$0104-07072011000200002

34. Alves MDFT, Carvalho DS, Albuquerque GSC. Barriers to patient safety incident reporting by brazilian health professionals: an integrative review. Cienc Saúde Coletiva. 2019;24(8):2895-908. https://doi.org/10.1590/1413-81232018248.23912017

35. Thomas L, Galla C. Building a culture of safety through team training and engagement. BMJ Qual Saf. 2013;22(5):425-34. https://doi.org/10.1136/bmjqs-2012-001011

36. Moreira FTLS, Callou RCM, Albuquerque GA, Oliveira RM. Estratégias de comunicação efetiva no gerenciamento de comportamentos destrutivos e promoção da segurança do paciente. Rev Gaúcha Enferm. 2019;40(esp):1-9. https: / /doi.org/10.1590/1983-1447.2019.20180308

37. Dias GC, Furegato ARF. Impacto do trabalho e satisfação da equipe multiprofissional de um hospital psiquiátrico. Rev Enferm Uerj. 2016;24(1):1-7. https://doi.org/10.12957/reuerj.2016.8164

38. Marinho MM, Radünz V, Rosa LM, Tourinho FSV, Itha $P$, Misiak M. Results of educational interventions on patient safety in error and adverse event reporting. Rev Baiana Enferm. 2018;32:1-12. 
39. Ubeda SRG. ¿Se necesita un esfuerzo para reemplazar la cultura punitiva por la de seguridad del paciente? Rev Calid Asist. 2016;31(3):173-6. https://doi.org/10.1016/j.cali.2015.09.007

40. Heidmann A, Trindade LF, Schmidt CR, Loro MM, Fontana RT, Kolankiewicz ACB. Contributive factors for the consolidation of patient safety culture in the hospital environment. Esc Anna Nery. 2020;24(1):1-8. https://doi.org/10.1590/2177-9465-EAN-2019-0153

41. Caldas BN, Portela MC, Singer SJ, Aveling EL. How can implementation of a large-scale patient safety program strengthen hospital safety culture? Lessons from a qualitative study of national patient safety program implementation in two public hospitals in Brazil. Med Care Res Rev. 2021;1-14. https://doi.org/10.1177/10775587211028068

42. Paim J, Travassos C, Almeida C, Bahia L, Maclnko J. The brazilian health system: history, advances, and challenges. Lancet. 2011;377(9779):1778-97. https://doi.org/10.1016/S0140-6736(11)60054-8
43. Ministério da Saúde (BR). Cadastro nacional de estabelecimentos de saúde (CNES). Brasília: Ministério da Saúde; 2020[acesso 29 out 2020]. Disponível em: http://tabnet.datasus.gov.br/cgi/tabcgi. exe?cnes/cnv/leiintbr.def

44. Negrelli B, Costa MAS, Abbas K, Galdamez EVC. Eficiência hospitalar nas regiões brasileiras: um estudo por meio da análise envoltória de dados. Rev Gest Sist Saúde. 2017;6(1):76-91. https://doi.org/10.5585/rgss.v6i1.314

45. Aveling EL, Kayonga Y, Nega A, Dixon-Woods M. Why is patient safety so hard in low-income countries? A qualitative study of healthcare workers' views in two African hospitals. Glob Health. 2015;11(1):4-11. https://doi.org/10.1186/s12992-015-0096-x

46. Dixon-Woods M, Baker R, Charles K, Dawson J, Jerzembek G, Martin $\mathrm{G}$ et al. Culture and behaviour in the english national health service: overview of lessons from a large multimethod study. BMJ Qual Saf. 2014;23(2):106-15. https://doi.org/10.1136/bmjqs-2013-001947

\section{Contribuição dos Autores}

Coslop S - Concepção, planejamento (desenho do estudo), aquisição, análise, interpretação dos dados e redação do trabalho. Portugal FB - Concepção, planejamento (desenho do estudo) e redação do trabalho. Pereira MSR - Aquisição, análise e interpretação dos dados. Calazans MSC - Análise e interpretação dos dados. Caldas BN - Análise, interpretação dos dados e redação do trabalho. Lima EFA - Redação do trabalho. Todos os autores aprovaram a versão final do trabalho.

\section{Conflito de Interesse}

Os autores informam não haver qualquer potencial conflito de interesse com pares e instituições, políticos ou financeiros deste estudo.

Licença CC BY-NC atribuição não comercial. Com essa licença é permitido acessar, baixar (download), copiar, imprimir, compartilhar, reutilizar e distribuir os artigos, desde que para uso não comercial e com a citação da fonte, conferindo os devidos créditos de autoria e menção à Visa em Debate. Nesses casos, nenhuma permissão é necessária por parte dos autores ou dos editores. 